\title{
Multiple Level Enhancement of Children's Picture Books with Augmented Reality
}

\author{
Nicholas Vanderschantz ${ }^{1}$, Annika Hinze ${ }^{1}$, and Aysha AL-Hashami ${ }^{2}$ \\ 1,2 University of Waikato, Hamilton, New Zealand \\ ${ }^{1}$ \{vtwoz, hinze\}@waikato.ac.nz ${ }^{2}$ assha1@students.waikato.ac.nz
}

\begin{abstract}
This paper reports a case study on using Augmented Reality (AR) in children's books. in which we explored the use of various types of interactions at different levels. An AR enhanced 2-page spread is developed to explore interactivity in printed books. We describe the design process and the insights gained into the requirements for AR enhancement of children's books.
\end{abstract}

Keywords: Augmented Reality, children's books, interaction model, reading.

\section{Introduction}

In this paper, we report on our case study into the opportunities of using Augmented Reality (AR) enhancement to printed children's picture books. The AR elements were developed by a design team following an established design process to identify the problem, hypothesize initial design ideations, iterate and develop design solutions. The paper focusses on the design decisions, reports on the proof-of-concept implementation, and discusses insights, however we do not discuss technical implementation of the AR.

\section{Related work}

It is well established that children may benefit from picture books through the consumption of both words and pictures (Bloom, 2002). We previously studied interactive children's books (Timpany \& Vanderschantz, 2012), identifying two types of interaction, physical interactions and intellectual interactions. Most interactive books feature either physical interaction or intellectual interaction, but few books cater for both forms of interaction (Timpany \& Vanderschantz, 2013).

Presently eBooks provide video, audio and interactive elements and some enhance engagement with the storyline through user in-book and out-of-book tasks. eBooks offer the opportunity for enhanced engagement with the storyline in ways that printed books cannot, yet this affordance has not been fully explored (Itzkovitch, 2012).

The potential of Augmented Reality integration on mobile devices has matured in recent years. AR has been explored for educational use (cf. Huang, Li and Fong, 2016) and AR books may assist children (cf. Yilmaz, Kucuk \& Goktas, 2017) and parents during shared reading of children's books (cf. Cheng \& Tsai, 2014). 


\section{$3 \quad$ Design Thinking}

We summarize here our design considerations for the AR application:

Focus on objects mentioned in the story text. The objects to animate in the AR view were decided to be those that were mentioned or described in the text of the story. The decision for each object depended on whether it was a primary object in the story or not. For example, the text in our example page explicitly mentions the panda, so it should be animated and pop-up when scanning the page. On the other hand, the tree is not mentioned in the text, and therefore was not animated.

Determining objects to be made visible or animated. While a number of inanimate objects can easily be shown on a page, fewer objects can be included in animations to avoid crowding on the phone screen. Having fewer animated objects was also deemed positive with regard to directing a child's attention and ensuring single focus.

Division of the target images. Each animation target must be unique and easy to recognize by the camera. It was decided not to encode any specific order for the animated objects to pop-up on the screen (but rather leave the selection to the reader).

Interaction types for different elements. It was decided to assign different types of interactions to the objects and to make these dependent on the element and the target reactions. We used both visual animations (appearing objects, movement) and sound animations (animal sounds, spoken text).

Camera level and distance. While children may hold the camera closer to the page when scanning the image, parents may trial different distances to the page to explore various levels. We decided to therefore make the required level of the camera flexible in order to suit different users. Integration of varied physical and intellectual interactivity was achieved through the unique use of multi-level targeting afforded by allowing different camera distances. Camera angle also needed to be considered to ensure correct identification of images irrespective of device orientation.

\section{$4 \quad$ Material Design}

We used the children's book Hannah's Favorite Place (Fiona Mason, 2015), a book written, illustrated, and developed as a traditional printed picture book, as well as an adaptive printed picture book (Wright, 2015), see example pages in Figure 1.
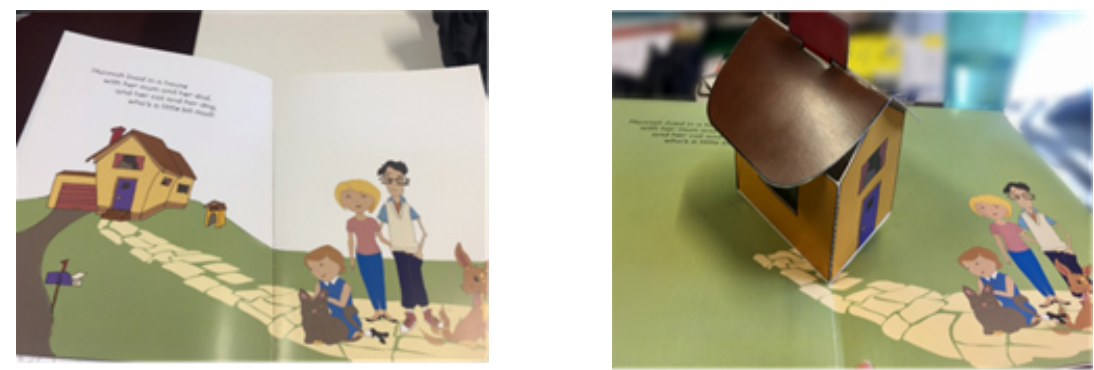

Fig. 1. Illustrations of the tradition (L) and Physically Enhanced (R) book. 


\subsection{Image Target}

For this investigation, we selected a single spread (i.e., two pages) showing one of Hannah's favorite places: the zoo. The text on this spread reads: "Her auntie took Hannah on trips to the zoo; they'd look at the pandas, the chimps and birds too" with a supporting illustration, some of which is mentioned in the text (see Figure 2).

The image target that was used for the AR enhancement converted some of the page elements into objects to be displayed in AR mode. Figure 3 shows the image target that was used in this project. This image target is similar to the spread found in the original version of the Hannah's favorite place, however some elements were initially hidden. Figure 2 shows the image as it first appears to a reader, while Figure 3 shows all animated objects. It can be seen that the following objects are initially hidden: the bird flying in front of the cat, the banana that the chimp was holding, as well as the drawing of the other chimp. These elements are discoverable through the use of AR interactions. Experiments were conducted to test all the possibilities of holding the camera by either the children or adults. We decided to divide the image into three levels as shown in Figure 3. The application has three levels of AR interactivity. These levels depend on how close the camera is when scanning the target. In each level, different animated objects aim to support both enjoinment and learning.

Level 1 (purple frame in Figure 3 - playful interactions): The user holds the camera close to the image target so the camera scans one object at a time (thus, there are six image targets in this level). In this level, the camera is closer to each object (assuming children will hold the phone). At this level, all objects were animated individually. A user can view and explore six primary objects (i.e. Hannah and her auntie, two pandas, two chimps, birds and a cat) on the target page. Each object will pop-up individually. Additionally, sounds will be played, such as screaming chimps when one of the chimps is being shown in the AR view. Figure 4 is a screenshot of the mobile app that shows an object from level one.

Level 2 (red frame in Figure 3 - simple educational interactions): The user holds the camera slightly further away from the image target so the camera can scan about one-third of the spread, i.e. two objects at a time. At this level, there are two image targets, and different objects appear in each part of the page. This level is educational but in a fun way aiming to assist children to learn how to spell words and recognize the related object of that name. As shown in the phone screen in Figure 5, this level introduces words and arrows that point to existing objects in the target page.

Level 3 (yellow frame in Figure 3 - playful and educational interactions): The user holds the camera furthest away from the image target so the camera can scan the whole spread at one time. There is only one image target to be discovered. This level was implemented to target both the enjoyment and learning aspects. Children can explore new objects in this level, which are the bees that appear only at this level. Moreover, in level three the story text was played in the background, i.e. the children can hear a person reading the text for them. Introducing new objects in this level could increase the excitement of the children and motivate them to hear the attached audio. 


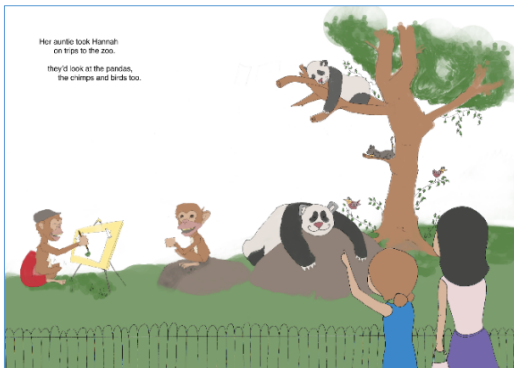

Fig. 2. Spread from Hannah's Favorite Place (Fiona Mason, 2015).

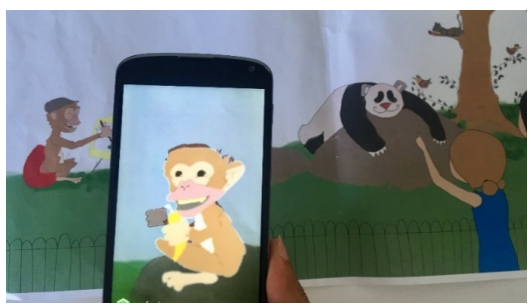

Fig. 4. An object that appears in level one.

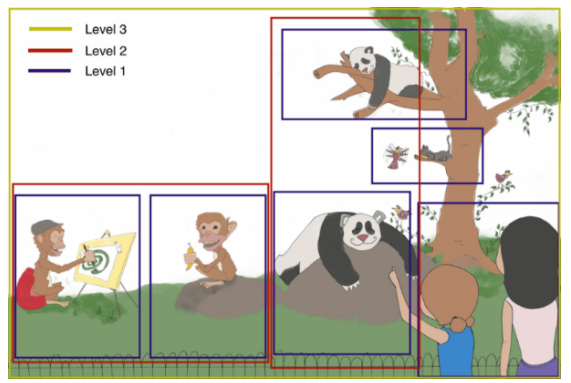

Fig. 3. The three levels of the image target.

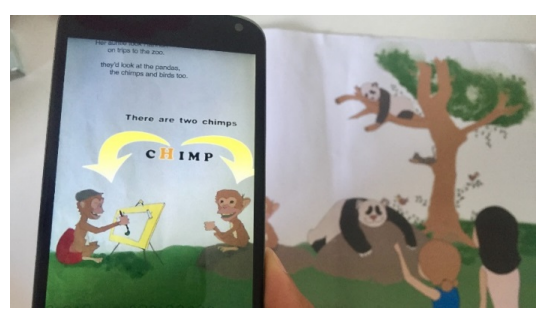

Fig. 5. Shows the word and arrows that appear in level two.

\section{Conclusions \& Recommendations}

The aim of our project was to develop an AR application for a children's picture book that explored the potential to engage both physical and intellectual interactions. We used three levels of interactivity to provide a variety of interactions and engagement opportunities. Our application combines fun and learning aspects at different camera levels. We engage the user in a range of physical and intellectual interactions through different types of animation and user interaction with the page. We used sounds, animated objects, as well as appearing and disappearing objects which we believe will draw children's attention and delight. Revealing objects like the banana and the bird in the application aims to increase children's engagement. Adding new objects (such as bees and additional text) in the augmented animations attempts to encourage children to explore the target page further. Using a number of enhancements in the AR version of the book should appeal to children's sense of curiosity, encouraging children to compare the two versions of the book (physical book and AR application), and afford opportunities to explore the book in more detail and through multiple sittings.

Further exploration of the possibilities of AR enhanced picture books at multiple interaction levels is warranted through user studies and development of further testing material. We extend this paper with reporting of insights from an initial expert walkthrough of this system in our related working paper (Vanderschantz et al., 2018). 


\section{References}

1. Bloom, P.: How Children Learn the Meanings of Words. MIT Press, Cambridge, MA, USA (2002).

2. Cheng, K.-H., Tsai, C.-C.: Children and parents reading of an augmented reality picture book: Analyses of behavioral patterns and cognitive attainment. Computers and Education, 72, 302-312 (2014).

3. Huang, Y., Li, H., \& Fong, R.: Using augmented reality in early art education: A case study in Hong Kong kindergarten. Early Child Development and Care, 186(6), 879-894 (2016).

4. Itzkovitch, A.: Interactive eBook Apps: The Reinvention of Reading and Interactivity, http://uxmag.com/articles/interactive-ebook-apps-the-reinvention-of-readingandinteractivity, (2012).

5. Timpany, C., Vanderschantz, N.: A Categorisation Structure for Interactive Children's Books. Int. J. Book. 9, 97-110 (2012).

6. Timpany, C., Vanderschantz, N.: Using a Categorisation Structure to Understand Interaction in Children's Books. Int. J. Book. 10, 29-44 (2013).

7. Yilmaz, R. M., Kucuk, S., \& Goktas, Y.: Are Augmented Reality Picture Books Magic or Real for Preschool Children Aged Five to Six. British Journal of Educational Technology, 48(3), 824-841 (2017).

8. Wright, K.: A comparison of children's books: Picture books versus physically and intellectually adaptive children's books. Unpublished Master's Thesis, University of Waikato, New Zealand (2015).

9. Vanderschantz, N., Hinze, A., \& Al-Hashami, A.: Using Augmented Reality to Enhance Children's Books. (Computer Science Working Papers 02/2018). Hamilton, New Zealand: Department of Computer Science, The University of Waikato. (2018). 